\title{
The Experiment of Acoustic Levitation and the Analysis by Simulation
}

\author{
Fuqiang Zhang, Zelai Jin
}

School of Mechanical and Automotive Engineering, Shanghai University of Engineering Science, Shanghai, China

Email: 1360199980@qq.com

How to cite this paper: Zhang, F.Q. and Jin, Z.L. (2018) The Experiment of Acoustic Levitation and the Analysis by Simulation. Open Access Library Journal, 5: e4948. https://doi.org/10.4236/oalib.1104948

Received: September 27, 2018

Accepted: October 22, 2018

Published: October 25, 2018

Copyright ( 2018 by authors and Open Access Library Inc.

This work is licensed under the Creative Commons Attribution International License (CC BY 4.0).

http://creativecommons.org/licenses/by/4.0/

\section{c) (i) Open Access}

\begin{abstract}
An acoustic levitation test bench was built to successfully suspend a small ball of a certain density. It was found that the smaller the diameter and density of the suspended sample, the easier it is to suspend. The diameter of the sample should not be larger than $1 / 4-1 / 3$ of the wavelength of the acoustic wave. In addition, as the sound pressure increases, the sound flow also affects the stability of the acoustic levitation. Comsol was used to numerical simulation the spatial sound field, pressure field and particle velocity distribution of different suspension cavity heights, and the factors affecting the acoustic levitation capacity were analyzed.
\end{abstract}

\section{Subject Areas}

Mechanical Engineering

\section{Keywords}

Acoustic Levitation, Sound Pressure, Numerical Simulation

\section{Introduction}

Acoustic levitation technology is mainly used to simulate various effects in the space environment, and better solve people's increasingly high demands on the experimental environment. Although the space resources that people have obtained so far are still very limited, the ultrasonic levitation technology is a good direction to create a vacuum environment similar to gravity-free, can also solve many chemical pharmaceuticals, no container processing, liquid solidification, precision instrument processing and other aspects. Regarding suspension, people may hear the most maglev train. In fact, the physical methods for achieving object suspension are various, such as: pneumatic suspension, electromagnetic sus- 
pension, acoustic suspension, optical suspension, electrostatic suspension, superconducting suspension, etc. Acoustic suspension is the use of the force generated by sound waves to resist gravity and achieve object suspension. The acoustic suspension does not need to consider the nature of the suspended object. Theoretically, it can suspend particles of any density. It is the first time in the world to suspend tungsten with a density of up to $18.9 \mathrm{~g} / \mathrm{cm}^{3}[1]$. The maximum levitation capacity reported in the literature is lead with a suspension density of $11.3 \mathrm{~g} / \mathrm{cm}^{3}$ [2]. The acoustic suspension will be studied below.

\section{Theory of Acoustic Levitation}

Ultrasonic standing wave suspension is a high-frequency piston vibration generated by the radiation end of the ultrasonic transducer [3], forming a sound field in the medium, and placing a reflection end on the acoustic wave transmission path to adjust the radiation end face and the reflection end face of the ultrasonic transducer The distance between them is an integer multiple of half wavelength, and the ultrasonic waves are repeatedly superimposed in the sound field space to form a high-strength standing wave sound field. The object placed in the standing wave sound field is subjected to the radiated sound pressure, balances its own gravity and hangs up, as shown in Figure 1.

It is usually required that the size of the suspended object $\left(R_{s}\right)$ is much smaller than the half wavelength $(\lambda / 2)[4]$. In practical applications, the diameter of the sample is generally not more than $\lambda / 4-\lambda / 3$. The acoustic radiation pressure due to the standing wave It is much larger than the traveling wave [the former is proportional to $\left(R_{s} / \lambda\right)^{3}$, the latter is proportional to $\left.\left(R_{s} / \lambda\right)^{6}\right]$, so the acoustic suspension experiment generally adopts standing wave. Taking the plane standing wave as an example, for the shape like $p=$ the sound field of $p=p_{0} \cos (k z) \sin (\omega t)$, the acoustic radiation pressure [5] is $F=(5 / 6) \pi R_{s}^{3}\left(\omega / \rho_{f} c^{3}\right) p_{0}^{2} \sin (2 k z)$. Here, $\rho_{f}$ and $c$ are the air density and the sound velocity, respectively, $\omega$ and $\mathrm{k}$ are the angular frequency and the wave number. It can be seen that the acoustic radiation pressure is distributed along the fluctuation direction in the period of $\lambda / 2$, at the sound pressure node $(z=\lambda / 4,3 \lambda / 4,5 \lambda / 4, \cdots)$, the sound radiation pressure has the property of restoring force, that is, once the sample deviates from the equilibrium

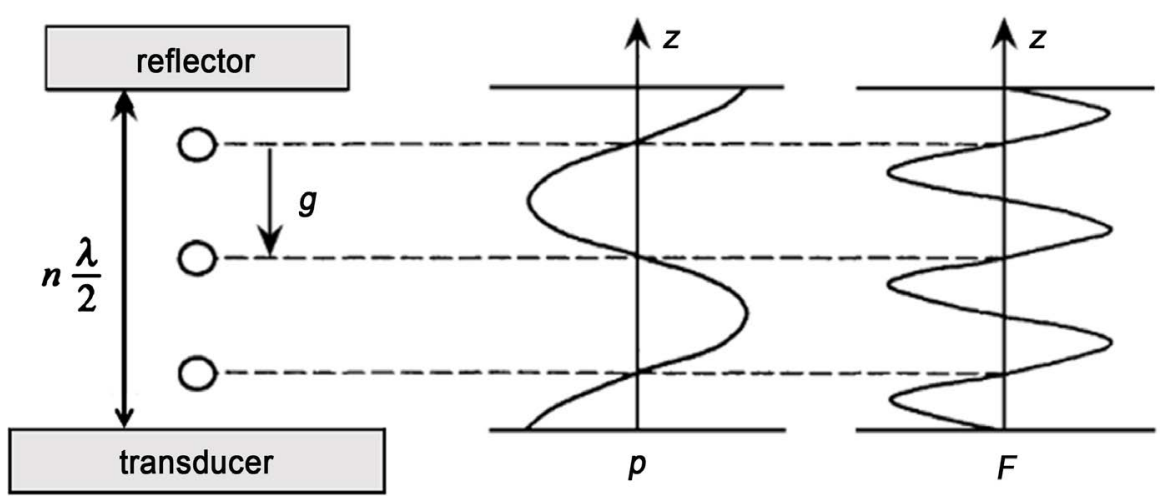

Figure 1. Acoustic suspension schematic. 
position, there is a tendency to be pulled back to the original position. These positions are the floating position of the sample. Usually, the sound is fluctuated. The direction is parallel to the direction of gravity to overcome the gravity of the sample. For heavier objects, the suspension position will be slightly below the sound pressure node. Experimental setup:

This paper selects $28 \mathrm{khz}$ ultrasonic transducer and generator circuit, $10 \mathrm{~mm}$ thickness reflection end, variable height longitudinal expansion platform, caliper, the particles used in the experiment are 2 - $3 \mathrm{~mm}$ polyethylene particles, the experimental results are as follows: white small particles uniform Suspended in the resonant cavity between the reflective end face and the ultrasonic transducer, the distance of the resonant cavity is $60 \mathrm{~mm}$, which is equal to 5 times the wavelength. The wavelength is calculated as the following Formula (1):

$$
\lambda=\frac{c}{f}
$$

The experimental results can be seen as shown in Figure 2. The distance between the white spheres is equal to $6 \mathrm{~mm}$. For the study of suspension stability, the numerical simulation analysis of the experiment is carried out. Barmatz and Collas are based on Gorkov's theory [6] and are used to evaluate various sound fields. The method of levitation force and stability of the suspended sample gives the average potential of the acoustic radiation force acting on the rigid ball in the ideal fluid:

$$
U=2 \pi R_{s}^{3}\left(\frac{\overline{P^{2}}}{3 \rho_{f} c^{2}}-\frac{\rho_{f} \overline{v^{2}}}{2}\right)
$$

\section{Simulation Analysis}

In this paper, the acoustic levitation force is simulated by the comsol software.

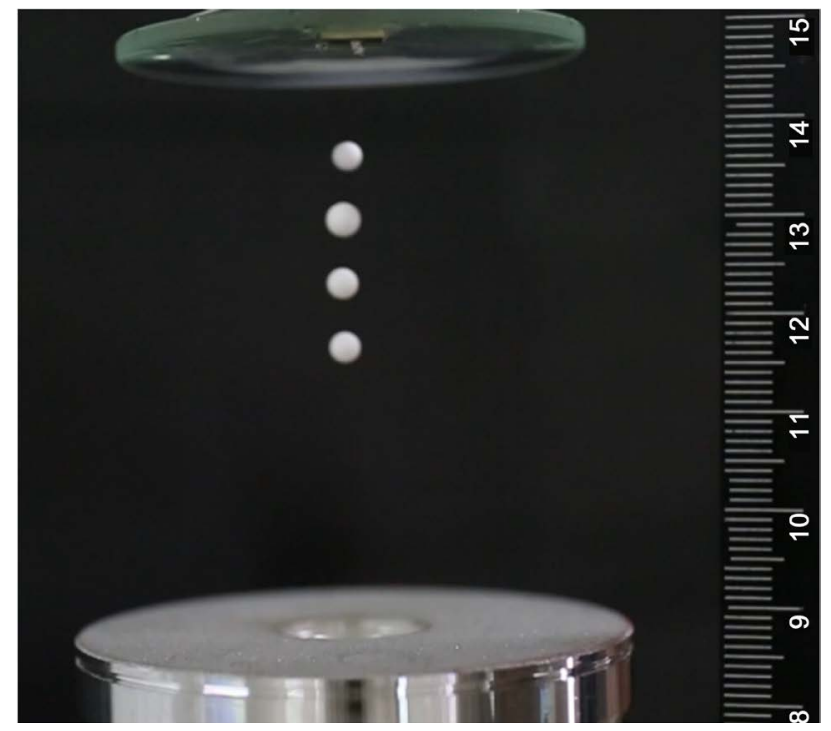

Figure 2. Acoustic suspension ball. 
The selected model is a two-dimensional simplified model of an acoustic levator driven at a constant frequency. Small elastic particles are translated in the acoustic standing wave field, and their motion paths are detected, which are affected by acoustic radiation force, viscous force, and gravity. The model requires particle tracking module parameters as shown in Table 1.

We want to study the distribution of acoustic levitation force, so we choose air particles model as the medium to characterize its distribution. In the calculation, we use dimensionless parameters. The dimensionless levitation force and restoring force coefficient are the quantities related only to the geometric parameters of the reflecting end face and the emitting end face. Therefore, the relationship between them and the geometric parameters are studied. To help us optimize the simulation analysis of the uniaxial suspension device, we calculated the potential energy of the air particle suspension position suspended at an integer multiple of half wavelength. The sound pressure equation used is as follows:

$$
\begin{gathered}
\nabla \cdot\left(-\frac{1}{\rho_{c}}\left(\nabla \rho_{t}-q_{d}\right)\right)-\frac{k_{e q}^{2} p_{t}}{\rho_{c}}=Q_{m} \\
p_{t}=p+p_{b} \\
k_{e q}^{2}=\left(\frac{\omega}{C_{c}}\right)^{2}-k_{z}^{2} \\
C_{c}=C, \rho_{c}=\rho \\
-n \cdot\left(-\frac{1}{\rho_{c}}\left(\nabla p_{t}-q_{d}\right)\right)=0
\end{gathered}
$$

The simulation results are as follows: the sound pressure in the middle position is the largest, which is in line with our experimental results, that is, the position

Table 1. Parameter settings.

\begin{tabular}{cccc}
\hline name & expression & value & description \\
\hline f0 & $28[\mathrm{kHz}]$ & $28000 \mathrm{~Hz}$ & Driving frequency \\
c0 & $343[\mathrm{~m} / \mathrm{s}]$ & $343 \mathrm{~m} / \mathrm{s}$ & Speed of sound \\
lambda0 & $\mathrm{c} 0 / \mathrm{f} 0$ & $0.01225 \mathrm{~m}$ & Wavelength \\
Dt & $2{ }^{*}$ lambda0 & $0.0245 \mathrm{~m}$ & Transducer diameter \\
Dr & $3^{*}$ lambda0 & $0.03675 \mathrm{~m}$ & Reflector diameter \\
H & $5^{*}$ lambda0/2 & $0.030625 \mathrm{~m}$ & Height \\
dvisc & $0.22\left[\mathrm{~mm}{ }^{*} \mathrm{sqrt}(100[\mathrm{~Hz}] / \mathrm{f} 0)\right.$ & $1.3148 \mathrm{E}-5 \mathrm{~m}$ & Viscous boundary layer thickness \\
d_p & lambda0/10 & $0.001225 \mathrm{~m}$ & Particle diameter \\
rho_p & $500\left[\mathrm{~kg} / \mathrm{m}^{3}\right]$ & $500 \mathrm{~kg} / \mathrm{m}^{3}$ & Particle density \\
rho_f & $1.2\left[\mathrm{~kg} / \mathrm{m}^{3}\right]$ & $1.2 \mathrm{~kg} / \mathrm{m}^{3}$ & Fluid density (air) \\
mu_f & $1.8 \mathrm{e}-5\left[\mathrm{~Pa}{ }^{*} \mathrm{~s}\right]$ & $1.8 \mathrm{E}-5 \mathrm{~Pa} \cdot \mathrm{s}$ & Fluid viscosity (air) \\
a0 & $1.5 \mathrm{e} 6\left[\mathrm{~m} / \mathrm{s}^{2}\right]$ & $1.5 \mathrm{E} 6 \mathrm{~m} / \mathrm{s}^{2}$ & Normal acceleration of transducer \\
\hline
\end{tabular}


of the suspended ball is in the blue position of the simulation, and the small ball particles suspended at the position have a pressure difference between the upper and lower sides. 4. Due to the powerful effect of the acoustic pressure [7], the suspended ball is restrained at this position due to the action of the sound pressure and is fixed at this point and suspended. In Figure 3, since the position of the transducer continuously emits ultrasonic waves at the bottom, the sound radiation pressure is correspondingly weakened upon returning.

Sound pressure level analysis [8] is also an important parameter to characterize the levitation capacity (Figure 4). The sound pressure level equation characterizes the distribution level of the levitation force. The force of the space ball is affected by different sound pressure levels, and the sound pressure level distribution map is simulated and analyzed. As shown in Figure 5 below, it is shown that the sound pressure level continuously changes in the resonant cavity, which is

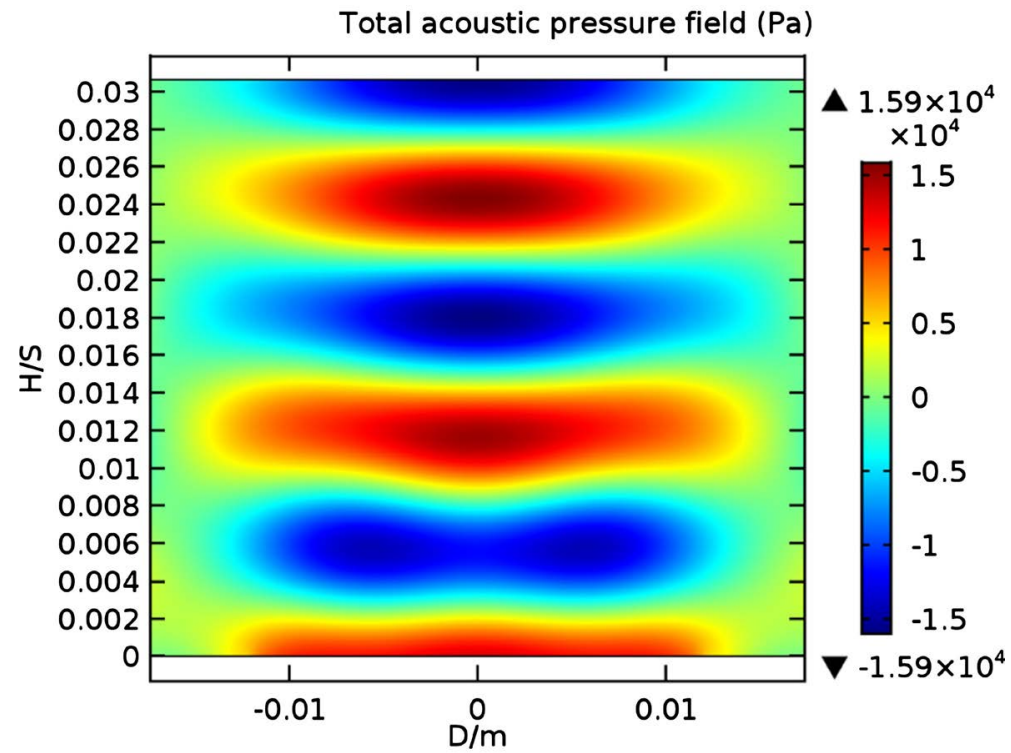

Figure 3. Total acoustic pressure.

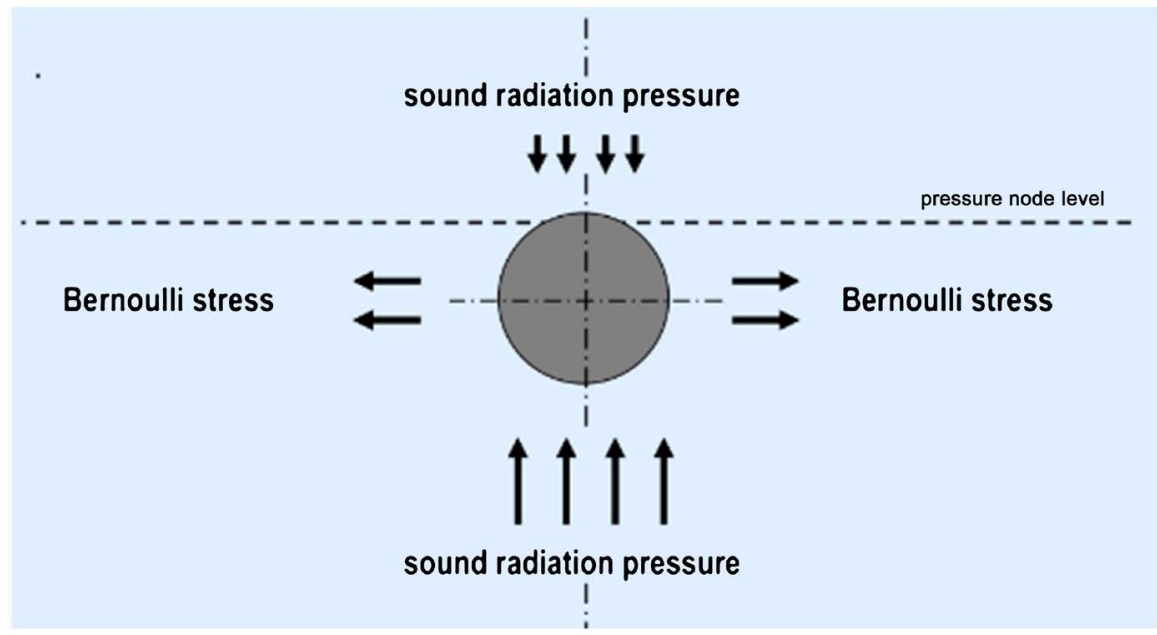

Figure 4. Particle in a sound pressure field. 
caused by the influence of particle vibration on the reflective end face, and the sound pressure level changes from high to low and then from low to high. The pressure level is the smallest, and the ball acting at this position will achieve suspension.

$$
P(y, t)=P_{\max } \cdot \cos (k \cdot y) \cdot \sin (\omega \cdot t)
$$

The acceleration of the simulated air particle plum movement is calculated by the acceleration formula of the sound pressure sound pressure level particle. The motion trajectory simulation is shown in Figure 6 below. It can be seen that the acceleration in the air density is dense due to the impact in the positive and

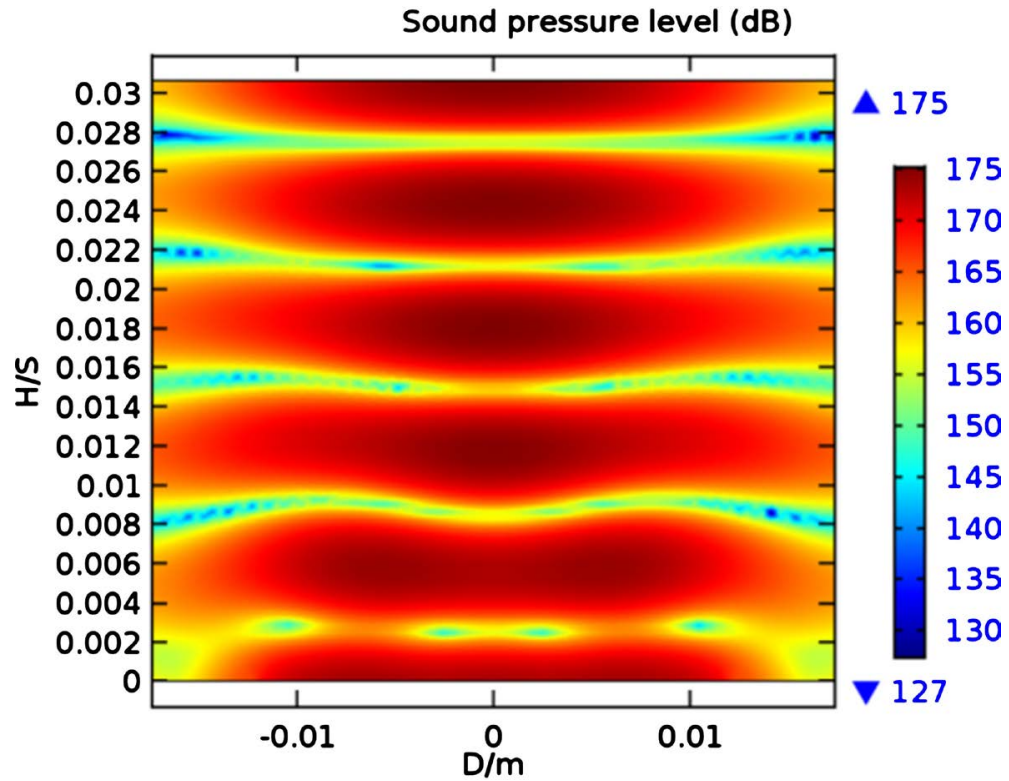

Figure 5. Sound pressure level.

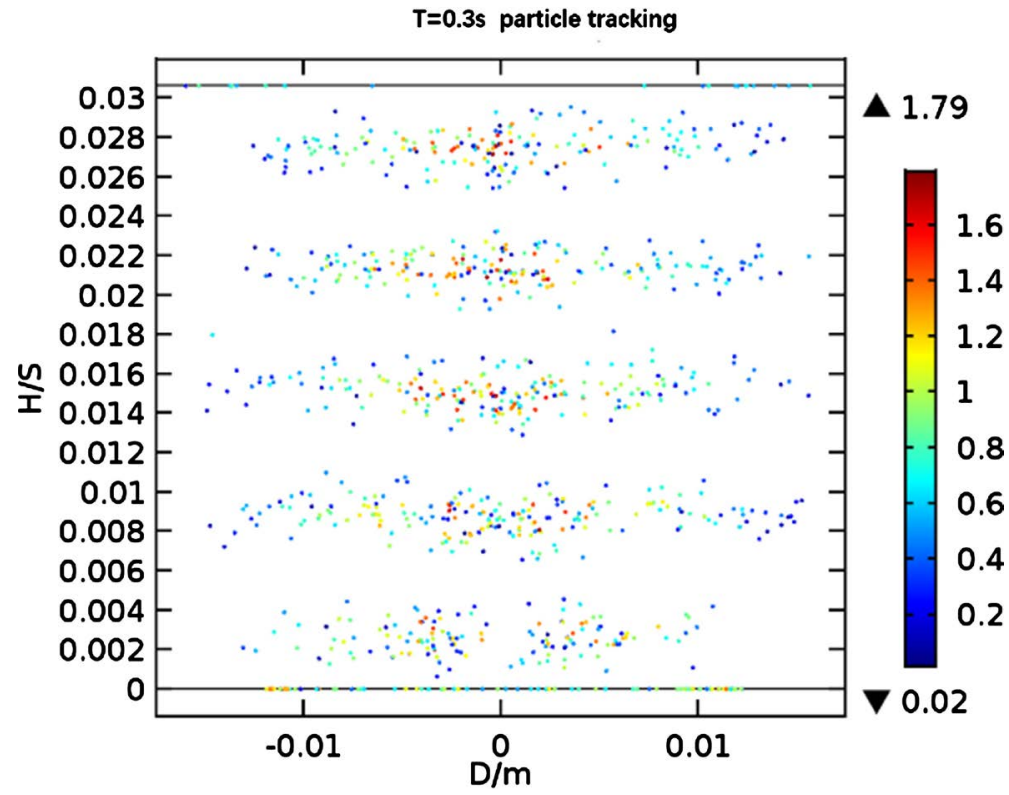

Figure 6. Particle tracking. 
negative directions. Offset each other, layered at half wavelength. Since the simulation calculates a dynamic process, the example reaches a stable stratification state when $0.3 \mathrm{~s}$, so $0.3 \mathrm{~s}$ is selected as the schematic diagram.

\section{Conclusion}

It is found through experiments that the suspended sample is affected by its own diameter and density. The diameter of the sample should not be larger than 1/4 $1 / 3$ of the wavelength of the acoustic wave. In addition, through simulation, it is concluded that with the increase of sound pressure, the sound flow will also affect the stability of sound suspension. The distribution of sound pressure level will change from large to small and then from small to large. The distribution trajectory provides the basis for future research.

\section{Conflicts of Interest}

The authors declare no conflicts of interest regarding the publication of this paper.

\section{References}

[1] Xie, W.J. and Wei, B. (2001) Parametric Study of Single-Axis Acoustic Levitation. Applied Physics Letters, 79, 881-883. https://doi.org/10.1063/1.1391398

[2] Deaver, L.E., et al. (2008) Temperature and Pressure Retrieval from Solar Occultation Measurements of Limb Path Transmittance and Refraction. AGU Spring Meeting AGU Spring Meeting Abstracts.

[3] Brandt, E.H. (2001) Acoustic Physics: Suspended by Sound. Nature, 413, 474. https://doi.org/10.1038/35097192

[4] Xie, W.J. (2002) Latest Progress in Acoustic Levitation. Physics, No. 9, 551-554.

[5] Yosioka, K. and Y. Kawasima. (1955) Acoustic Radiation Pressure on a Compressible Sphere. Acta Acustica United with Acustica, 5, 167-173.

[6] Gor'Kov, L.P. (1962) On the Forces Acting on a Small Particle in an Acoustical Field in an Ideal Fluid. Soviet Physics Doklady, 6, 773.

[7] Sharfarets, B.P., Kurochkin, V.E. and Knyaz'Kov, N.N. (2008) Radiation Pressure in an Arbitrary Incident Field: Relation to the Inclusion Scattering Amplitude. Doklady Physics, 53, 356-359. https://doi.org/10.1134/S1028335808070057

[8] Shaw, E.A. (1974) Transformation of Sound Pressure Level from the Free Field to the Eardrum in the Horizontal Plane. Journal of the Acoustical Society of America, 56, 1848-1861. https://doi.org/10.1121/1.1903522 